\title{
Contribuições do Paradigma de Equivalência de Estímulos para o Estudo das Atitudes
}

\author{
Tahcita Medrado Mizael \\ Universidade Federal de São Carlos \\ Silvana Lopes dos Santos \\ Universidade Federal de São Carlos \\ Júlio Cesar Coelho de Rose \\ Universidade Federal de São Carlos
}

\begin{abstract}
RESUMO
Considerando a abordagem analítico-comportamental, atitudes podem ser consideradas respostas avaliativas emitidas de acordo com uma história prévia de aprendizagem. $\mathrm{O}$ paradigma de equivalência de estímulos, utilizando procedimentos que simulam o comportamento simbólico no laboratório, tem possibilitado entender a formação de atitudes como uma rede de relações arbitrárias entre classes de estímulos e atributos avaliativos. Esta visão tem auxiliado na compreensão de como fenômenos sociais, tais como estereótipos e preconceitos, podem ser estabelecidos e modificados. O objetivo deste trabalho é apresentar algumas contribuições, a partir de evidências empíricas demonstradas por pesquisas que utilizaram este paradigma nas últimas décadas, particularmente a partir da década de 90, que fortalecem a equivalência de estímulos como um modelo comportamental importante para o estudo das atitudes.
\end{abstract}

Palavras-Chave: paradigma de equivalência de estímulos; atitudes; preconceito; estereótipos.

\begin{abstract}
Contributions of stimulus equivalence paradigm for studying attitudes

According to the behavior-analytic approach, attitudes can be viewed as responses emitted based on a previous learning history. Stimulus equivalence paradigm, using procedures that simulate symbolic behavior in the laboratory, has made possible to understand attitude formation as arbitrary relational networks between stimulus classes and evaluative attributes. This view has enabled understanding how social phenomena, such as prejudices and stereotypes, can be established and changed. The aim of this research is to present contributions, from empirical evidence demonstrated by stimulus equivalence research carried out in the last decades, especially from the 1990's, that strengthen this paradigm as an important behavioral model for studying attitudes.
\end{abstract}

Keywords: stimulus equivalence paradigm; attitudes; prejudice; stereotypes.

O conceito de atitudes tem sido considerado central na psicologia social, com um amplo campo de pesquisas e estudos (e.g. Allport, 1935; Schwarz \& Bohner, 2001). Definido, inicialmente, como algo amplo e composto por diversos componentes, como cognitivo, afetivo e comportamental (e.g. Schwarz \& Bohner, 2001), tal concepção perdeu parte dessa amplitude no decorrer dos anos, passando o conceito a ser definido basicamente a partir de seu componente avaliativo (e.g. Bohner \& Dickel, 2011; Maio \& Haddock, 2009).

As definições de Allport (1935) e de Eagly e Chaiken (1993) exemplificam essa mudança. Allport define atitudes como "um estado mental e neural de prontidão (readiness), organizado a partir da experiência, exercendo uma influência diretiva e dinâmica sobre as respostas de indivíduos a todos os objetos e situações com as quais se relaciona" (p. 810). Décadas depois, a atitude é definida como "uma tendência psicológica que é expressada pela avaliação de determinada entidade, com algum grau de aprovação ou desaprovação" (Eagly \& Chaiken, 1993, p. 1).

Apesar das mudanças nas concepções, uma característica que sempre esteve presente, de maneira aberta ou encoberta nas definições, era o caráter estável e du- 
radouro das atitudes, e seu relacionamento com os comportamentos dos indivíduos (Schwarz \& Bohner, 2001). Essa visão das atitudes como algo durável e, principalmente, constante, começou a mudar quando diversos estudos passaram a sugerir que as atitudes não eram tão estáveis e duradouras como tradicionalmente presumido (e.g. Schwarz, 2007). Outros estudos mostraram, inclusive, que a relação próxima entre atitudes e comportamento, também suposta nas definições mais tradicionais, só era demonstrada em contextos específicos e sob condições estritas (e.g. Tourangeau \& Rasinski, 1988; Wicker, 1969).

Uma das áreas que tem demonstrado a maleabilidade das atitudes, assim como sua não-correspondência fiel e constante com comportamentos, é a análise do comportamento. Fundamentada pela filosofia do behaviorismo radical, essa área da psicologia entende que a emissão de respostas, públicas ou privadas, depende de uma história prévia que aumente ou diminua a probabilidade da emissão de tais respostas, de modo que as consequências de comportamentos passados "selecionem" quais comportamentos serão emitidos no futuro. Veja que, primeiramente, para essa área, as atitudes não são simplesmente consideradas relacionadas aos comportamentos, mas são comportamento (aberto ou encoberto), emitido ou evocado de acordo com uma história prévia de aprendizagem. Guerin (1994), por exemplo, se refere às atitudes como comportamento verbal, estando assim sob controle das contingências sociais. Neste sentido, elas podem assumir diferentes funções (como tato, mando, etc.), a depender das contingências de reforçamento de uma comunidade verbal. Este conceito permite, portanto, que a formação e mudança de atitudes sejam investigadas a partir das relações que são estabelecidas com o ambiente e não como algo que é causado por instâncias "dentro da pessoa".

Muitas pesquisas nos últimos anos têm investigado as atitudes tendo como base os princípios do condicionamento clássico. Normalmente, o foco destes trabalhos é a mudança da resposta avaliativa de um estímulo, também chamado Evaluative Conditioning (EC) (ver De Houwer, Thomas, \& Baeyens, 2001 para uma revisão; Hofmann, de Houwer, Perugini, Baeyens, \& Crombez, 2010; Sweldens, van Osselaer, \& Janiszewski, 2010). O procedimento padrão nesta área consiste em parear de maneira sistemática um estímulo avaliado como neutro a outro com valência positiva ou negativa, de modo que a resposta avaliativa ao estímulo neutro se modifique, tornando-se positiva ou negativa, a depender do estímulo utilizado (Levey \& Martin,1975; Staats \& Staats, 1957).
Um estudo clássico dessa área foi realizado por Levey e Martin (1975). A pesquisa teve a participação de 10 adultos voluntários com desenvolvimento típico, com idades entre 21 a 40 anos, os quais categorizaram cartões com imagens ou fotografias não familiares em três grupos: "gosto" (liked), "neutro" (neutral) e "não gosto" (disliked). Após isto, eles também deveriam escolher, dentro de cada categoria, dois cartões de que mais gostavam e dois dos quais menos gostavam. As figuras da categoria neutra eram escolhidas pelo experimentador de acordo com a similaridade de forma, conteúdo e cor. Após esta fase, os cartões eram arranjados em cinco pares na seguinte sequência: neutro-gosto, neutro-não gosto, neutro-neutro, não gosto-neutro $\mathrm{e}$ gosto-neutro. $\mathrm{O}$ participante era instruído a pressionar um botão que desencadeava a apresentação sucessiva de cada par de figuras em ordem randomizada. Após a apresentação dos estímulos, o participante deveria categorizar as figuras em "gosto" e "não gosto" e, além disto, as mesmas deveriam ser arranjadas em ordem de preferência. Os resultados apontaram que os cartões neutros adquiriram a valência daqueles aos quais foram pareados, o que foi mais acentuado quando os estímulos cuja valência era considerada positiva ou negativa foram apresentados após os estímulos considerados neutros. Este procedimento ficou conhecido como "paradigma figura-figura".

Apesar de estas pesquisas indicarem que o pareamento direto entre um estímulo considerado neutro e outro com valência positiva ou negativa é suficiente para que a mudança de avaliação ocorra, este pareamento direto entre um estímulo com significado e um neutro não parece ser um pré-requisito para que o mesmo fenômeno seja observado. Uma abordagem que também tem sido utilizada para estudar as atitudes é o paradigma de equivalência de estímulos (Sidman \& Tailby, 1982; Sidman, 1994). Este paradigma consiste em uma maneira de analisar, no laboratório, como estímulos sem similaridade física tornam-se substituíveis entre si, em algumas ocasiões. Um dos procedimentos amplamente utilizados para se estabelecer relações arbitrárias entre estímulos tem sido o emparelhamento de acordo com o modelo (ou matching-to-sample - MTS, em inglês). Um estímulo modelo é apresentado juntamente com dois ou mais estímulos de comparação. Diante de um estímulo-modelo, apenas um dos estímulos de comparação é considerado correto, estabelecendo-se assim uma relação condicional entre dois estímulos. Por exemplo, se A1 é apresentado, então escolho B1; se A2 é apresentado, então escolho B2, e assim por diante. Os estímulos usados são frequentemente figuras, sílabas ou 
palavras sem sentido, e são convencionalmente designados por combinações alfanuméricas; nas pesquisas relevantes para o estudo de atitudes, contudo, estímulos significativos são frequentemente utilizados, em conjunto com estímulos sem significado. Após o treino direto de, pelo menos, dois conjuntos de relações (por exemplo, A1 com B1 e também com $\mathrm{C} 1$, e A2 com B2 e também com $\mathrm{C} 2$, estabelecendo as relações designadas como A1B1, A1C1, A2B2 e A2C2), nos quais os participantes recebem feedback informativo sobre seus acertos e erros, são realizados testes para verificar a emergência de relações entre estímulos que não foram estabelecidas diretamente. Essas relações, denominadas emergentes ou derivadas, podem atestar as propriedades de reflexividade, simetria e transitividade, indicando que as relações entre os estímulos são relações de equivalência e os estímulos relacionados entre si constituem uma classe de estímulos equivalentes, ou seja, substituíveis entre si (em algumas ocasiões) no controle do comportamento. A reflexividade é verificada pela relação estabelecida entre um estímulo e ele mesmo (e, frequentemente, é assumida para indivíduos verbais e não verificada explicitamente). A simetria é constatada a partir da bidirecionalidade da relação entre estímulos (e.g. após ser ensinado a escolher B1 diante de A1, ser capaz de escolher A1 diante de B1 sem a necessidade de ensino explícito) e, por fim, a transitividade é constatada pela emergência da relação entre dois estímulos distintos, relacionados entre si por um terceiro (e.g. se $\mathrm{AB}$ e $\mathrm{AC}$, então $\mathrm{BC}$ ). Os testes que combinam simetria e transitividade, neste exemplo $\mathrm{CB}$, são muitas vezes chamados de testes de equivalência porque evidenciam, simultaneamente, a emergência de duas propriedades das relações de equivalência. Esse paradigma constitui, portanto, uma valiosa contribuição para a psicologia, uma vez que amplia as análises existentes sobre a geratividade dos comportamentos, e oferece um modelo para o estudo de comportamentos simbólicos (de Rose \& Bortoloti, 2007).

Vários trabalhos têm demonstrado também o que tem sido chamado de transferência de funções ${ }^{1}$, isto é, certas funções comportamentais de um estímulo podem ser transferidas para (ou melhor, compartilhadas com) os demais membros da classe (e.g., Amd, Barnes-Holmes, \& Ivanoff, 2013; Barnes-Holmes, Barnes-Holmes, Smeets e Luciano, 2004; de Rose, Mcllvane, Dube, Galpin \& Stoddard, 1988; Dougher, Augustson, Markham, Greenway, \& Wulfert, 1994; Wulfert \& Hayes, 1988). Isto ocorre sem a necessidade de ensino direto, ou seja, apenas pela participação em uma classe de equivalência, de modo que "um estímulo que tem determinadas funções pode ser comparado a um 'referente', e os estímulos equivalentes a ele podem ser comparados a 'símbolos' capazes de substituí-los em algumas ocasiões” (de Rose \& Bortoloti, 2007, p. 253).

Neste contexto, considerando a visão analítico-comportamental, é possível entender as atitudes como a avaliação de pessoas, objetos e eventos, mesmo na ausência de qualquer experiência direta com tais estímulos. Como observou Biglan, não se pode ignorar a importância de focalizar o grau de resposta favorável ou desfavorável a um estímulo, e "behavior analysts would ignore this literature at their peril" (Biglan, 1995, p. 82). Deste modo, o objetivo deste artigo é apresentar algumas contribuições do paradigma de equivalência de estímulos como uma das possíveis maneiras de se estudar a formação e mudança de atitudes, apresentando, especificamente, uma metodologia que pode ser útil para estudar esse assunto. Inicialmente serão descritos alguns trabalhos que utilizaram este paradigma para formar atitudes e preferências. Em seguida, serão relatadas pesquisas da área cujos objetivos têm sido a reversão ou mudança de atitudes preconceituosas, vieses e estereótipos. Por fim, serão feitas considerações apontando a importância destes estudos para a compreensão de fenômenos sociais e de como o paradigma de equivalência de estímulos pode ser uma abordagem útil neste processo.

\section{Equivalência de estímulos e formação de atitudes}

Pesquisas que utilizam o paradigma de equivalência de estímulos têm demonstrado que funções condicionadas classicamente podem ser transferidas nas relações de equivalência, gerando, por exemplo, respostas de medo e ansiedade a estímulos os quais a pessoa não teve uma experiência direta anterior (ver Dougher, 1998, para uma revisão). Um exemplo é a pesquisa realizada por Dougher, Augustson, Markham, Greenway, \& Wulfert (1994, estudo 1) com 8 universitárias, as quais foram remuneradas por sua participação e também poderiam receber bônus contingente ao desempenho nas tarefas. $\mathrm{O}$ procedimento consistiu inicialmente na formação de duas classes de estímulos (símbolos abstratos) com quatro membros (A1B1C1D1 e A2B2C2D2), utilizando tentativas de MTS. A segunda fase do estudo envolvia um condicionamento clássico discriminativo no qual um membro de uma classe servia como estímulo condicional positivo (CS+) e um membro da outra classe como negativo (CS-). Um choque elétrico de baixa intensidade (selecionado para cada participante) servia como estímulo incondicionado (US). A tarefa operante de cada participante era pressi- 
onar a barra cada vez que um símbolo aparecesse na tela do computador, o que lhe acarretava pontos. Quando B1 aparecia na tela, a participante recebia um choque, mas não quando B2 aparecia. A análise dos níveis de condutância da pele das participantes demonstrou que, para sete delas, houve diferença de resposta de eliciação na presença de todos os estímulos da classe 1 (relacionada com o choque), em comparação com os da classe 2 (sem relação com o choque). Sem qualquer treino direto, foi observada, portanto, a transferência de função de eliciação do medo (indicada pelos níveis de condutância da pele) para estímulos que nunca foram emparelhados diretamente com choque, mas que eram equivalentes a um estímulo submetido a tal emparelhamento.

Barnes-Holmes et al. (2004) fizeram uma pesquisa semelhante na qual verificaram que funções de humor, como tristeza e alegria, também podem ser transferidas via equivalência de estímulos. Neste experimento, 16 voluntárias na faixa de 19 a 30 anos de idade (10 universitárias e seis não-universitárias) foram treinadas a formar duas classes de equivalência com três membros cada (A1B1C1 e A2B2C2), cujos estímulos eram sílabas sem sentido. Após esta fase, duas músicas clássicas, avaliadas como eficazes para a indução de humor feliz ou triste, foram tocadas na presença de um membro de cada classe (a música para o humor feliz foi tocada diante de $\mathrm{B} 1$, e a música para o humor triste diante de B2). Depois deste procedimento de indução verificouse, utilizando escalas de humor, se as participantes avaliariam os estímulos B1 e B2 de acordo com a música correspondente. $\mathrm{O}$ teste de transferência de funções consistia na avaliação dos estímulos $\mathrm{C} 1$ e $\mathrm{C} 2$ (que não passaram pela indução de humor) pelas mesmas participantes. Estes estímulos induziram as mesmas funções de humor dos elementos da classe a que pertenciam, ou seja, C1 foi avaliado como induzindo sentimentos de felicidade e $\mathrm{C} 2$ de tristeza.

Na mesma linha, Bortoloti e de Rose (2007, 2009, 2011, 2012) têm desenvolvido várias pesquisas nas quais expressões faciais humanas são adotadas como estímulos significativos, as quais já teriam por si só um efeito de eliciar respostas emocionais. Após a formação de classes, símbolos que antes não tinham nenhum significado especial, ou seja, que eram considerados neutros, passam a ser avaliados de forma semelhante às faces. Essa transferência de funções entre estímulos equivalentes tem sido validada por várias medidas independentes, como o diferencial semântico (Bortoloti \& de Rose, 2007, 2009, 2012; de Almeida \& de Rose, 2015), o Implicit Relational Assessment Procedure
(IRAP; Bortoloti \& de Rose, 2012), o priming semântico (Barnes-Holmes et al., 2005; Bortoloti \& de Rose, 2011), e medidas de potencias relacionados a eventos (PREs) (Barnes-Holmes et al., 2005; Bortoloti, Pimentel \& de Rose, 2014; Haimson, Wilkinson, Rosenquist, Ouimet, \& McIlvane, 2009), o que demonstra a generalidade deste fenômeno.

Grey e Barnes (1996, estudo 1) realizaram um estudo particularmente interessante sobre formação de atitudes, por inserir estímulos com significado pré-experimental. A pesquisa teve a participação de 11 universitários voluntários (5 homens e 7 mulheres). Em um de seus experimentos, após a formação de classes com três estímulos (A1B1C1 e A2B2C2), formadas por sílabas sem sentido, cada um dos participantes assistiu a dois vídeos curtos, um dos quais dramatizava cenas de sexo (rotulado com a sílaba sem sentido B1) e o outro cenas religiosas (rotulado com a sílaba sem sentido B2). A escolha dos vídeos foi feita com base em um estudo anterior que mostrou que indivíduos que avaliaram a sexualidade de modo positivo avaliaram a religiosidade de modo negativo, e vice-versa.

Após esta fase, em um dos estágios do experimento, os participantes tinham que categorizar tanto estes vídeos (B1 e B2) quanto outros não assistidos, mas rotulados com as outras sílabas previamente utilizadas durante a fase de treino (A1, C1, A2 e C2) como "bons" ou "ruins" (sem consequências diferenciais para tais respostas). Os resultados mostraram que ocorreu transferência de funções para todos os participantes, ou seja, eles classificaram os vídeos não vistos na mesma categoria que o vídeo assistido de acordo com a classe estabelecida: os participantes que classificaram as cenas de sexo como boas, classificaram os vídeos A1 e C1 como bons, e os vídeos $\mathrm{A} 2$ e $\mathrm{C} 2$ como ruins, e viceversa. Este estudo demonstrou, portanto, como é possível formar atitudes sem a necessidade de uma história direta entre os estímulos, ou seja, apenas pela participação nas classes de equivalência.

Seguindo esta mesma abordagem, alguns trabalhos têm também verificado que escolhas e preferências por determinado produto, como refrigerantes e alimentos, podem ser influenciadas pelas relações de equivalência (Arntzen, Fagerstrom \& Foxall, 2016; Barnes-Holmes, Keane, Barnes-Holmes \& Smeets, 2000; Smeets \& Barnes-Holmes, 2003; Santos \& de Rose, submetido; Straatmann, Almeida \& de Rose, 2014).

Smeets e Barnes-Holmes (2003, experimento 1), por exemplo, investigaram a escolha e preferência por refrigerantes de 16 crianças com desenvolvimento típico, 
na faixa dos cinco anos de idade. Foi realizado um treino de MTS com o objetivo de formar duas classes de estímulos, sendo que, em uma delas o estímulo significativo era a figura do Ernie (um personagem amigável conhecido), e na outra a figura de uma criança chorando. Os demais estímulos eram figuras geométricas e símbolos abstratos. O teste de transferência de funções consistiu em oferecer às crianças duas garrafas iguais com o mesmo refrigerante. A única diferença é que em uma delas o rótulo era o símbolo pertencente à classe do personagem infantil, e na outra, o símbolo pertencente à classe da criança chorando. Era perguntado então qual refrigerante a criança gostaria de provar primeiro, e, depois de provar os dois refrigerantes, de qual ela havia gostado mais. Os resultados indicaram que 14 das 16 crianças escolheram provar primeiro o refrigerante cujo rótulo pertencia à classe da figura preferida, e 12 deles demonstraram preferência pela mesma bebida. Tais resultados replicaram os encontrados em um estudo anterior com estudantes universitários, utilizando palavras emotivas ao invés de figuras (Barnes-Holmes et al., 2000).

Nos dois estudos supracitados, os participantes provavam os alimentos, no entanto, resultados semelhantes podem ser encontrados quando se investiga apenas a preferência declarada ou avaliativa. Straatmann et al. (2014, experimento 2) criaram nomes de alimentos fictícios (capira, fulito e piteba) para verificar a formação de preferência alimentar em adolescentes com desenvolvimento típico, na faixa de 11 a 14 anos de idade, utilizando o paradigma de equivalência de estímulos. Com o uso de escalas e testes de preferência, 36 estudantes de escola pública (18 meninos e 18 meninas), avaliaram estes alimentos tendo acesso somente aos seus nomes. Em seguida, cada adolescente participou de um treino de MTS, visando a formação de três classes de estímulos, onde cada uma delas continha uma expressão facial esquemática (alegre, triste ou neutra), símbolos abstratos, e o nome de um dos três alimentos. Os testes de preferência foram realizados novamente e houve uma diferença significativa entre a avaliação obtida inicialmente e a final. Como esperado, o alimento fictício capira, que pertencia a classe da face feliz, teve uma avaliação mais positiva, enquanto que o alimento piteba, pertencente à classe da face triste, foi avaliado mais negativamente.

\section{Mudança de atitudes, estereótipos e preconceito}

Como visto na seção anterior, a formação de atitudes pode ser conceituada como a formação de classes de equivalência entre uma classe de estímulos (e.g. um grupo social, um conjunto de objetos) e atributos avaliativos. A partir de tal conceituação, seria possível então reverter ou modificar algumas atitudes por meio da reversão ou reorganização de classes de equivalência? Algumas pesquisas tiveram o intuito de investigar estas questões (e.g. McGlinchey \& Keenan, 1997; Moxon, Keenan \& Hine, 1993; Watt, Keenan, Barnes, Cairns, 1991), mostrando a efetividade da análise do comportamento na compreensão de temas socialmente sensíveis, como o preconceito. No estudo realizado por Dixon e Lemke (2007), por exemplo, os autores definiram operacionalmente o que é costumeiramente chamado de "viés", "estereótipo", ou "preconceito" como o "responder relacional direcionado a membros de uma raça específica" (p. 5). Com o objetivo de alterar avaliações negativas de pessoas do Oriente Médio, e avaliar uma possível transferência de funções após submeter os participantes a um treino com o intuito de reverter tais classificações, os autores recrutaram 12 universitários norte-americanos (brancos ou afro-americanos) para a pesquisa, que receberam créditos extras de curso pela participação.

Inicialmente, foi feito um pré-teste, onde os participantes tinham que avaliar, em uma escala Likert que variava entre 1 - mau (evil) e 10 - bom (good), imagens de homens do Oriente Médio, de homens americanos e de objetos. Nessa fase, nove fotografias não utilizadas no treino foram inseridas, com o intuito de verificar uma possível transferência de funções no pós-teste. A fase de treino consistiu em ensinar os participantes a relacionar, inicialmente, símbolos abstratos (A1, A2 e A3) com as palavras bom (good - B1), neutro (neutral B2) e mau (evil - B3), e, em seguida, os mesmos símbolos do conjunto A com fotografias de homens do Oriente Médio (C1), de objetos (C2), e de homens americanos (C3). Após o treino, testes das relações derivadas de simetria e equivalência foram realizados, e por fim foi feito o pós-teste, de maneira idêntica ao préteste.

Como esperado, os participantes avaliaram as fotografias de homens do Oriente Médio com valores inferiores aos atribuídos aos homens norte-americanos durante o pré-teste. Após demonstrarem as relações de simetria e de equivalência, contudo, a maioria dos participantes reverteu suas classificações dadas no pósteste, avaliando os americanos com valores inferiores aos atribuídos aos homens do Oriente Médio. Além disso, os resultados evidenciaram transferência de funções para a maioria dos participantes. Na discussão, os autores sugerem que uma rede relacional de equivalência entre atos terroristas, indivíduos terroristas e carac- 
terísticas da cultura do Oriente Médio pode ter sido formada, especialmente tendo em vista os acontecimentos de 11 de setembro de 2001 e sua repercussão, demonstrando, portanto, como o preconceito com relação à tal população pode ter sido estabelecido para os participantes norte-americanos.

O trabalho realizado por de Carvalho e de Rose (2014) teve como objetivo examinar o nível de interferência de relações pré-experimentais a respeito de atitudes raciais na formação de classes de equivalência por crianças. Inicialmente, 16 crianças com desenvolvimento típico e idades entre 7 e 10 anos classificaram fotos de pessoas brancas e negras com atributos que poderiam variar entre as esferas positiva e negativa. As crianças que atribuíram valores inferiores a faces negras, em comparação com as faces brancas, realizaram ainda um segundo teste para confirmar o viés racial obtido no primeiro instrumento. Neste segundo teste, as crianças deveriam escolher, diante de faces negras (C1), estímulos abstratos (C2) ou faces brancas (C3), uma mão fazendo um símbolo de positivo (A1) ou a mesma mão fazendo um sinal de negativo com o polegar (A2). As crianças que, após apresentarem um responder estável, revelassem um viés negativo para as faces negras, em comparação com as faces brancas, foram recrutadas para a pesquisa.

As quatro crianças recrutadas ( 3 meninos e uma menina) receberam treino das relações $A B$ e, em seguida, $\mathrm{BC}$, sendo que os estímulos da classe $\mathrm{B}$ eram símbolos abstratos, e os estímulos $\mathrm{C} 3$ não foram utilizados na fase de treino. $\mathrm{O}$ teste de equivalência realizado (CA), idêntico ao apresentado no pré-teste, foi diferente dos testes tradicionais, uma vez que inseriu, em todas as tentativas, uma das faces brancas $(\mathrm{C} 3$, que não foi utilizado durante o treino) como um terceiro estímulo-modelo. Nesse estudo, apenas uma criança reverteu as relações pré-experimentais, passando a relacionar as faces de negros com os estímulos positivos, e deste modo, demonstrando as classes de equivalência previstas pelo procedimento de treino.

Estudos como esses, que fizeram uma ponte entre o paradigma de equivalência de estímulos e atitudes preconceituosas e/ou estereotípicas, mostram os alcances da análise do comportamento diante de temas socialmente relevantes, mas, ao mesmo tempo, evidenciam um possível limite: a não formação das classes de equivalência para grande parte dos participantes nas pesquisas que utilizam estímulos com significado pré-experimental, especialmente quando tais estímulos são considerados "socialmente sensíveis". Tem sido ar- gumentado que a dificuldade em formar tais classes de equivalência ocorre porque há uma interferência, uma vez que as relações pretendidas pelos experimentadores são conflitantes com as relações pré-experimentais (e.g. Haydu, Camargo, \& Bayer, 2015; Haydu, Gaça, Cognetti, Costa, \& Tomanari, 2015; Moxon et al., 1993; Straatmann et al., 2014; Watt et al., 1991).

Mizael, de Almeida, Silveira e de Rose (2016) denominaram tal delineamento, no qual o treino das relações $\mathrm{AB}$ e $\mathrm{BC}$ levam à potencial formação de classes de equivalência conflitantes de "Conflicting Relations $\mathrm{Pa}$ radigm" (CRP). Levando em consideração que, na história de vida dos indivíduos, as relações pré-experimentais são amplamente reforçadas pela comunidade verbal à qual os sujeitos pertencem, é muito provável que um treino de aproximadamente uma ou duas horas, como o que é lado aos participantes dessas pesquisas, seja insuficiente para modificar tais relações.

Uma exceção foi o estudo de Mizael et al. (2016), no qual todos os participantes formaram as classes de equivalência previstas pelos experimentadores. Neste estudo, 13 crianças ( 8 meninas e 5 meninos, 7 brancas e 5 negras) com desenvolvimento típico e idades entre 8 a 10 anos, que demonstraram viés racial negativo para faces negras em um pré-teste, receberam uma versão modificada do treino utilizado por de Carvalho e de Rose (2014), de modo a formar classes de equivalência entre faces de pessoas negras e um símbolo considerado positivo (mão fazendo o símbolo de positivo com o polegar). Todas as crianças formaram as classes de equivalência, e houve transferência da função positiva para as faces negras, de modo que a diferença estatisticamente significativa entre as avaliações de faces negras e brancas encontrada no pré-teste se mostrou não significativa no pós-teste. Desse modo, a suposta limitação deste paradigma pode ser devida (pelo menos em parte) a questões metodológicas. A pesquisa de Mizael et al. utilizou alguns parâmetros de treino e de testes que têm sido apontados como facilitadores tanto para a formação, quanto para a reorganização de classes de equivalência, como o uso do protocolo simples para o complexo e a redução gradual da consequenciação das respostas durante a última fase de treino, como forma de preparação para os testes, o que provavelmente contribuiu para os efeitos observados. Isso não significa que não possa existir interferência entre as classes préexperimentais e as classes "conflitantes" previstas pelos experimentadores, mesmo quando os participantes formam as classes de equivalência. No estudo de Mizael et al. (2016), em um teste de equivalência modificado, no qual faces negras, estímulos abstratos e faces brancas 
serviram como comparações, apesar de todos os participantes terem previamente relacionado as faces negras com o símbolo positivo, quatro participantes passaram a relacionar as faces brancas com o símbolo positivo, mostrando que as relações pré-experimentais podem ser fonte de conflito com relações aprendidas experimentalmente.

\section{Por que estudar a formação e mudança de atitudes com o paradigma de equivalência de estímulos?}

O objetivo deste trabalho foi apontar algumas contribuições do paradigma de equivalência de estímulos para o entendimento e estudo das atitudes. As pesquisas supracitadas mostraram como estímulos com uma valência neutra podem passar a ser considerados positivos ou negativos, a partir de emparelhamentos indiretos. Da mesma forma, foi possível constatar também que estímulos considerados inicialmente positivos ou negativos podem ter seus graus de valência modificados e/ou revertidos, após uma breve história de treino (ou seja, um treino cuja duração varie entre minutos e poucas horas). Tais constatações sugerem que a avaliação de objetos, pessoas e eventos pode emergir a partir de uma rede de relações arbitrárias e não somente por meio da experiência direta com tais estímulos. Assim, relações entre estímulos (por exemplo, $\mathrm{AB}$ e $\mathrm{AC}$ ) podem ser ampliadas pela emergência de relações não-treinadas (como $\mathrm{BA}, \mathrm{CA}, \mathrm{BC}$ e CB), assim como pela transferência de funções (se A1 tem uma função discriminativa ou eliciadora, B1 e C1 provavelmente também terão tal função), mostrando o potencial gerativo deste modelo, ou seja, a existência de relações que não podem ser explicadas por meio do ensino direto. Esse caráter gerativo tem implicações, por exemplo, ao se estudar formas de preconceito/atitudes que se originaram, a despeito de não terem tido uma história direta de reforçamento (ver, por exemplo, Guerin, 1994).

Outra contribuição deste paradigma pode ser descrita pelo termo economia de ensino, uma vez que não é necessário ensinar cada uma das relações para que um indivíduo as aprenda. Pensando especificamente nas atitudes, foco deste estudo, imagine uma criança que aprendeu diretamente as relações mulher-magra, magra-bonita, e bonita-feliz. Enquanto o treino informaria à criança que "mulheres têm que ser magras", "pessoas magras são bonitas", e "pessoas bonitas são felizes", a formação das classes implicaria na noção de que "mulheres magras são bonitas e felizes", e que "mulheres bonitas e felizes são magras", revelando como este modelo pode contribuir para o estudo de diversos estereótipos e preconceitos existentes nas sociedades.
A flexibilidade das relações é outra característica com implicações em diversas questões, como o preconceito, o humor, e as preferências alimentares. Duas preocupações bastante comuns entre pais são o que fazer para seus filhos se alimentarem melhor, e como seus filhos declaram não gostar de certos alimentos que eles nunca experimentaram. $\mathrm{O}$ paradigma de equivalência de estímulos pode auxiliar tanto na explicação de como essa relação (entre alimentos saudáveis e atributos negativos) pode ter sido estabelecida, como em sugerir estratégias para tentar revertê-la, de modo a fazer crianças ingerirem (e declararem atitudes mais positivas a) alimentos mais saudáveis.

Ainda relacionado a esta questão, os estudos descritos sobre escolha e preferência de alimentos demonstram o quanto estas relações derivadas podem estar presentes também nas estratégias de marketing. Tais estudos têm contribuído para apontar como o uso de figuras positivas, como personagens, podem influenciar as escolhas e preferências por uma "marca", mesmo que os consumidores nunca tenham consumido determinados produtos. A transferência de funções positivas pelo uso de um personagem conhecido, ou de uma celebridade, por exemplo, em comerciais de produtos pode ser utilizada para entender a "explosão" nas vendas, especialmente quando a marca é nova ou é lançado um novo produto de uma marca reconhecida (e.g. Arntzen et al., 2016).

Outro ponto que relaciona a questão da flexibilidade com as atitudes sociais é o que alguns pesquisadores das ciências sociais se referem como "ressignificação", que seria descrita como a apropriação, ou seja, o uso de termos considerados pejorativos para descrever grupos minoritários por esses próprios indivíduos, de maneira a "esvaziar" seu sentido pejorativo, e visibilizar tais grupos. Movimentos sociais, como os LGBTs, por exemplo, são conhecidos por terem ressignificado termos como "queer", "viado", e "sapatão" (queer, embora signifique "estranho", "esquisito", foi e ainda é utilizado de modo pejorativo para descrever indivíduos cuja identidade ou performance de gênero e sexualidade não são hegemônicos. "Viado" e "sapatão" são igualmente utilizados como forma de insultar gays e lésbicas, respectivamente - e.g. Butler, 1993; Facchini, 2005). No movimento negro, por exemplo, também houve a ressignificação dos termos "raça" e black (e.g. "black power").

Ainda para exemplificar, um indivíduo que é gay e começa a se autorreferir como "viado", pode promover mudanças nas relações inicialmente estabelecidas entre 
"viado" e "ruim", e entre "ruim" e "desprezível", por exemplo, uma vez que outras relações, como entre o próprio indivíduo (que é "viado") e "cidadão comum", e entre "cidadão comum" e "bom" podem ser formadas. Desse modo, a relação "viado-cidadão comum-bom" pode emergir, assim como a transferência das funções de "bom", para o homem gay que se autodenomina "viado", revertendo a relação "viado-ruim", ou seja, a atitude preconceituosa. Esse mesmo raciocínio pode ser aplicado ao termo raça, que foi ressignificado de modo a revelá-lo como uma construção social. Uma explicação comportamental para o fenômeno da "ressignificação" pode ser, portanto, a formação de novas relações de equivalência entre palavras que descrevam indivíduos ou grupos e atributos avaliativos neutros ou positivos, que poderia ocorrer pela reorganização ou reversão das classes de equivalência originalmente criadas, e transferência de funções "positivas" para essas palavras: veja que a relação "viado-ruim" e "ruim-desprezível", por exemplo, pode ser modificada a partir do estabelecimento das relações supracitadas. Deste modo, o paradigma de equivalência de estímulos pode contribuir com o entendimento de fenômenos descritos em outras literaturas, como o fenômeno da ressignificação, previamente descrito, ao fornecer maneiras cientificamente demonstradas pelas quais a ressignificação, ou modificação dessas atitudes, pode ocorrer. Assim, este paradigma é capaz de auxiliar tanto no entendimento de como certas atitudes podem ser estabelecidas, como na sugestão de possíveis intervenções comportamentais para atitudes consideradas preconceituosas e/ou que possam gerar danos físicos e/ou psicológicos aos indivíduos envolvidos em certas categorias avaliativas negativas.

Por fim, especificar condições nas quais novas habilidades podem emergir a partir de um treino, é outro ponto forte deste paradigma, e de grande valia para a ciência, que preza pela previsão e controle das variáveis estudadas. As pesquisas supracitadas são apenas um recorte de uma área que, embora não seja extensa como a da psicologia social, tem conseguido dados importantes e sistemáticos sobre uma maneira pela qual a formação e a mudança de atitudes pode ser conceituada. Ou seja, embora o paradigma de equivalência de estímulos seja considerado um modelo comportamental que permita o estudo da formação e mudança de atitudes, cabe ressaltar que tal paradigma não é o único conjunto de saberes da análise do comportamento que têm se dedicado ao estudo dessa temática.

Como previamente mencionado, o condicionamento clássico, chamado também de evaluative conditioning em alguns contextos, é outra forma de entender a formação e mudança de atitudes, a partir da exposição de estímulos com valência positiva ou negativa a estímulos neutros, de maneira simultânea. Entretanto, dados têm demonstrado que seus efeitos são breves e elusivos (e.g. de Houwer, Baeyens \& Field, 2005; Jones, Olson \& Fazio, 2010). Também tem sido utilizado para o estudo da formação e mudança de atitudes o condicionamento do tipo respondente. Este é um procedimento semelhante ao utilizado no condicionamento clássico, mas que difere devido a apresentação de vários estímulos condicionados em várias sequências, assim como o comportamento produzido por tal procedimento, que é considerado majoritariamente operante (Leader, Barnes-Holmes, \& Smeets, 2000; Leader \& Barnes-Holmes, 2001). Os estudos empregando este procedimento (e.g. Smyth, Barnes-Holmes, \& Forsyth, 2006; Tyndall, Roche, \& James, 2009) mostram que ele permite verificar a transferência de funções.

Guerin (1994) também apresenta uma interpretação comportamental das atitudes, conceituando-as como comportamento verbal, especificamente, com as funções de tato (descrever, por exemplo, gostar ou não de um estímulo com o qual se teve uma experiência direta), intraverbal (atitudes controladas por comportamento verbal, como quando alguém diz "não gosto de índios", por ter somente lido que determinada tribo é "selvagem"), e mando (como quando uma pessoa em uma posição de poder diz "não gosto de pessoas que me pedem favores"). As atitudes seriam, portanto, controladas pelas contingências sociais em vigor em determinada sociedade, e não simplesmente descrições de eventos privados, como tradicionalmente descrito pela psicologia social.

Por fim, pesquisadores da teoria das molduras relacionais (RFT, em inglês - Hayes, Barnes-Holmes \& Roche, 2001) têm estudado fenômenos que vão além da formação de classes de equivalência, como descrita pelo paradigma de equivalência de estímulos. Desse modo, outras relações, como hierarquia e oposição, por exemplo, podem também emergir a partir de um treino no qual duas ou mais relações são ensinadas, e as funções atribuídas a um ou mais estímulos de uma classe são transformadas de acordo, nos outros estímulos da classe. Dixon, Dymond, Rehfeldt, Roche e Zlomke (2003) oferecem alguns exemplos de como várias relações podem emergir, no contexto do terrorismo: imagine um jovem do Oriente Médio que está com dificuldades financeiras graves, passando fome inclusive (A). Essa condição evoca sentimentos de indignação (B), que serão transferidos para a "América", após o 
jovem ouvir um grupo de homens da sua cidade dizendo que a América é culpada pela pobreza desses povos (C). Por enquanto, a análise pode facilmente ser entendida por meio de uma relação de equivalência (ou coordenação, na terminologia da RFT) entre A, B e C. Agora, imagine que este jovem vê que algumas pessoas de seu povoado (exército Talibã) têm condições financeiras favoráveis, não passam fome e tem esposas. Essa condição favorável $(\mathrm{Y})$ é oposta à condição à qual o jovem se encontra (A). Também opostos aos sentimentos de indignação dele (B), são os sentimentos evocados por uma promessa de dinheiro e possibilidade de ter uma esposa (Z). Assim, se A é o oposto de Z, e se A é oposto de $Y$, então $Z$ é igual a $Y$, o que significa que o jovem pode acreditar que entrar no exército Talibã trará felicidade e dinheiro. A teoria das molduras relacionais, portanto, parece ampliar as possibilidades de entendimento das atitudes, como entendido pelo paradigma de equivalência de estímulos, o que justifica a necessidade de mais pesquisas dessa área, relacionando, inclusive, as relações de equivalência (ou coordenação) a outras relações.

Concluindo, o paradigma de equivalência de estímulos, embora não seja a única forma de analisar as atitudes, oferece aos estudiosos da análise do comportamento e de outras áreas, maneiras de se conceber como as atitudes podem ser formadas e modificadas, sem que haja uma história de aprendizagem direta, a partir de procedimentos bem estabelecidos na literatura, os quais têm obtido resultados sistemáticos e condizentes com os fenômenos observados. Isso mostra que essa área deve ser expandida, por permitir a análise de uma série de atitudes, especialmente àquelas intimamente relacionadas com o preconceito e os estereótipos (raciais, religiosos, de gênero, étnico, etc.), os quais podem ter consequências negativas para indivíduos ou grupos de indivíduos que são estigmatizados com base em informações que nem sempre são acuradas, e que, inclusive, podem estar relacionadas sem um contato direto com tais grupos ou indivíduos. Isto reforça o quanto estes estudos podem trazer contribuições sociais importantes, especialmente quando diferentes áreas passam a dialogar, aumentando o escopo e o alcance das pesquisas, e auxiliando no desenvolvimento de estratégias para intervenções mais efetivas. Embora o campo de pesquisas relacionando este paradigma à formação e mudança de atitudes ainda esteja em desenvolvimento, este trabalho objetivou evidenciar, entre outras coisas, seu caráter promissor para o entendimento de como certas atitudes podem ser formadas e modificadas. Espera-se que isto também possa levar à formula- ção de intervenções que, de fato, sejam benéficas para diversas populações que são estigmatizadas cotidianamente.

\section{REFERÊNCIAS}

Allport, G. W. (1935). Attitudes. In C. Murchison (Ed.), Handbook of Social Psychology. Worcester, Mass: Clark University Press.

Amd, M., Barnes-Holmes, D., \& Ivanoff, J. (2013). A derived transfer of eliciting emotional functions using differences among electroencephalograms as a dependent measure. Journal of the Experimental Analysis of Behavior, 99, 318-334. doi:10.1002/jeab.19.

Arntzen, E., Fagerstrom, A., \& Foxall, G. (2016). Equivalence classes and preferences in consumer choices. In G.R. Foxall (Ed.), The Routledge Companion to Consumer Behavior Analysis. (pp. 65-77). Hove, UK: Routledge.

Barnes-Holmes, Y., Barnes-Holmes, D., Smeets, P. M., \& Luciano, C. (2004). A derived transfer of mood functions through equivalence relations. The Psychological Record, 54, 95-04.

Barnes-Holmes, D., Keane, J., Barnes-Holmes, Y., \& Smeets, P. M. (2000). A derived transfer of emotive functions as a means of establishing differential preferences for soft drinks. The Psychological Record, 50, 493-511.

Barnes-Holmes, D., Staunton, C., Whelan, R., BarnesHolmes, Y., Commins, S., Walsh, D., \& Dymond, S. (2005). Derived stimulus relations, semantic priming, and event-related potentials: Testing a behavioral theory of semantic networks. Journal of the Experimental Analysis of Behavior, 84, 417-433. doi: 10.1901/jeab.2005.78-04

Bennett, M. P., Meulders, A., Baeyens, F., \& Vlaeyen, J. W. (2015). Words putting pain in motion: The generalization of pain-related fear within an artificial stimulus category. Frontiers in Psychology, 6, 1-12. doi:10.3389/fpsyg.2015.00520

Biglan, A. (1995). Changing cultural practices. A contextualist framework for intervention research. Reno, NV: Context Press.

Bohner, G., \& Dickel, N. (2011). Attitudes and attitude change. Annual Review of Psychology, 62, 391-417. doi: 10.1146/annurev.psych.121208.131609

Bortoloti, R., \& de Rose, J. C. (2007). Medida do grau de relacionamento entre estímulos equivalentes. Psicologia: Reflexão e Crítica, 20, 252-258.

Bortoloti, R., \& de Rose, J. C. (2009). Assessment of the relatedness of equivalent stimuli through a semantic differential. The Psychological Record, 59, 534-590.

Bortoloti, R., \& de Rose, J. C. (2011) An "Orwellian" Account of Stimulus Equivalence: Are Some Stimuli "More Equivalent" Than Others? European Journal of Behavior Analysis, 12, 121-134.

Bortoloti, R., \& de Rose, J. C. (2012). Equivalent stimuli are more strongly related after training with delayed matching than after simultaneous matching: 
A study using the Implicit Relational Assessment Procedure (IRAP). The Psychological Record, 62(1), 41-54.

Bortoloti, R., Pimentel, N., \& de Rose, J. C. (2014). Electrophysiological investigation of the functional overlap between semantic and equivalence relations. Psychology \& Neuroscience, 7(2), 183-191. http://dx.doi.org/10.3922/j.psns.2014.015

Butler, J. (1993). Bodies that matter: On the discursive limits of "sex". New York: Routledge.

de Almeida, J. H., \& de Rose, J. C. (2015). Changing the meaningfulness of abstract stimuli by the reorganization of equivalence classes: Effects of delayed matching, The Psychological Record, 65, 451-461. doi:10.1007/s40732-015-0120-9

de Carvalho, M. P. de., \& de Rose, J. C. (2014). Understanding racial attitudes through the stimulus equivalence paradigm. The Psychological Record, 64, 527-536. doi: 10.1007/s40732-014-0049-4

De Houwer, J., Baeyens, F., \& Field, A. P. (2005). Associative learning of likes and dislikes: Some current controversies and possible ways forward. Cognition and Emotion, 19, 161-174. doi: 10.1080/02699930441000265

De Houwer, J., Thomas, S., \& Baeyens, F. (2001). Associative learning of likes and dislikes: A review of 25 years of research on human evaluative conditioning. Psychological Bulletin, 127, 853-869. doi: 10.1037/0033-2909.127.6.853

de Rose, J. C., \& Bortoloti, R. (2007). A equivalência de estímulos como modelo de significado. Acta Comportamentalia, 15(3), 83-102.

de Rose, J. C., McIlvane, W. J., Dube, W. V., Galpin, V. C., \& Stoddard, L. T. (1988). Emergent simple discrimination established by indirect relation to differential consequences. Journal of the Experimental Analysis of Behavior, 50(1), 1-20. doi: 10.1901/jeab.1988.50-1

Dixon, M. R., \& Lemke, M. (2007). Reducing prejudice towards Middle Eastern persons as terrorists. European Journal of Behavior Analysis, 8(1), 5-12.

Dixon, M. R., Dymond, S., Rehfeldt, R. A., Roche, B., \& Zlomke, K. R. (2003). Terrorism and Relational Frame Theory. Behavior and Social Issues, 12, 129147.

Dougher, M. J. (1998).Stimulus equivalence and the untrained acquisition of stimulus functions. Behavior Therapy, 29, 577-591. doi: 10.1016/S00057894(98)80018-X

Dougher, M. J., Augustson, E., Markham, M. R., Greenway, D. E., \& Wulfert, E. (1994). The transfer of respondent eliciting and extinction functions through stimulus equivalence classes. Journal of the Experimental Analysis of Behavior, 62 (3), 331-351. doi: 10.1901/jeab.1994.62-331

Eagly, A. H., \& Chaiken, S. (1993). The nature of attitudes. In: A. H. Eagly, \& S. Chaiken, (Eds.), The Psychology of attitudes. (pp. 1-21). Belmont: Fort Worth.

Facchini, R. (2005). Sopa de letrinhas: Movimento homossexual e produção de identidades coletivas nos anos 1990. Rio de Janeiro: Garamond.

Grey, I. M., \& Barnes, D. (1996). Stimulus equivalence and attitudes. The Pshycological Record, 46, 243270.

Guerin, B. (1994). Attitudes and beliefs as verbal behavior. The Behavior Analyst, 17(1), 155-163.

Haimson, B., Wilkinson, K. M., Rosenquist, C., Ouimet, C., \& McIlvane, W. J. (2009). Electrophysiological correlates of stimulus equivalence processes. Journal of the Experimental Analysis of Behavior, 92(2), 245-256. doi: 10.1901/jeab.2009.92-245

Haydu, V. B., Camargo, J., \& Bayer, H. (2015). Effects of preexperimental history on the formation of stimulus equivalence classes: A study with supporters of Brazilian soccer clubs. Psychology \& Neuroscience, 8(3), 385-396. http://dx.doi.org/10.1037/h0101276

Haydu, V. B., Gaça, L. B., Cognetti, N. P., Costa, C. E., \& Tomanari, G. Y. (2015). Equivalência de estímulos e ciúme: Efeito de historia pré-experimental. Psicologia: Reflexão e Crítica, 28(3), 490-499.

Hayes, S. C., Barnes-Holmes, D., \& Roche, B. (Eds.). (2001). Relational Frame Theory: A post-Skinnerian account of human language and cognition. New York: Plenum Press.

Hofmann, W., de Houwer, J., Perugini, M., Baeyens, F., \& Crombez, G. (2010). Evaluative conditioning in humans: A meta-analysis. Psychological Bulletin, 136(3), 390-421. doi: 10.1037/a0018916

Jones, C. R., Olson, M. A., \& Fazio, R. H. (2010). Evaluative conditioning: The "how" question. Advances in Experimental Social Psychology, 1(43), 205-255. doi: 10.1016/S0065-2601(10)43005-1

Leader, G., \& Barnes-Holmes, D. (2001). Matching-tosample and respondent-type training as methods for producing equivalence relations: Isolating the critical variable. The Psychological Record, 51, 429444.

Leader, G., Barnes-Holmes, D., \& Smeets, P. M. (2000). Establishing equivalence relations using a respondent-type procedure III. The Psychological Record, 50, 63-78.

Levey, A. B., \& Martin, I. (1975). Classical conditioning of human 'evaluative' responses. Behavior Research and Therapy, 13, 221-26. http://dx.doi.org/10.1016/0005-7967(75)90026-1

Maio, G., \& Haddock, G. (2009). The Psychology of attitudes and attitude change. Sage Publications. Wales.

McGlinchey, A., \& Keenan, M. (1997). Stimulus equivalence and social categorization in Northern Ireland. Behavior and Social Issues, 7, 2, 113-128. http://dx.doi.org/10.5210/bsi.v7i2.310

Mizael, T. M., de Almeida, J. H., Silveira, C. C., \& de Rose, J. C. (2016). Changing racial bias by transfer of functions in equivalence classes. The Psychological Record, 66, 451-462. doi: 10.1007/s40732-0160185-0

Moxon, P., Keenan, M., \& Hine, L. (1993).Gender-role stereotyping and stimulus equivalence. The Psycho- 
logical Record, 43, 381-394.

Santos, S. L., \& de Rose, J. C. (2016). Food Marketing to Children: Investigating the Impact of Stimulus Equivalence on Children's Food Choice. Manuscrito submetido para publicação.

Schwarz, N. (2007). Attitude construction: Evaluation in context. Social Cognition, 25, 638-656.

Schwarz, N., \& Bohner, G. (2001). The construction of attitudes. In A. Tesser, \& N. Schwarz (Eds.), Blackwell Handbook of Social Psychology: Intrapersonal processes. (pp. 436-457). Oxford, UK: Blackwell.

Sidman, M. (1994). Equivalence relations and behavior: A research story. Boston, MA: Authors Cooperative.

Sidman, M., \& Tailby, W. (1982). Condi $\neg$ tional discrimination vs. matching to sample: An expansion of the testing para $\neg$ digm. Journal of the Experimental Analysis of Behavior, 37, 5-22. doi: 10.1901/jeab.1982.37-5

Smeets, P. M., \& Barnes-Holmes, D. (2003). Children's emergent preferences for soft drinks: Stimulus-equivalence and transfer. Journal of Economic Psychology, 24, 603-618. http://dx.doi.org/10.1016/S0167-4870(03)00004-7

Smyth, S., Barnes-Holmes, D., \& Forsyth, J. P. (2006). Derived transfer of simple discrimination and selfreported arousal functions in spider fearful and nonspider-fearful participants. Journal of the Experimental Analysis of Behavior, 85(2), 223-246. doi: 10.1901/jeab.2006.02-05

Staats, C. K., \& Staats, A. W. (1957). Meaning established by classical conditioning. Journal of Experimental Psychology, 54, 74-80. http://dx.doi.org/10.1080/00221309.1963.9920556
Straatmann, G., Almeida, S. S., \& de Rose, J. C (2014). Computerized assessment of food preferences in adolescents in the stimulus equivalence paradigm. Trends in Psychology, 22(3), 613-624. doi: 10.9788/TP2014.3-07

Sweldens, S. T. L. R., van Osselaer, S. M. J., \& Janiszewski, C. (2010). Evaluative conditioning procedures and the resilience of conditioned brand attitudes. Journal of Consumer Research, 37(3), 473-489. http://dx.doi.org/10.1086/653656

Tourangeau, R., \& Rasinski, K. A. (1988). Cognitive processes underlying context effects in attitude measurement. Psychological Bulletin, 103(3), 299-314. doi: 0033-2909/88/\$00.75

Tyndall, I. T., Roche, B., \& James, J. E. (2009). The interfering effect of emotional stimulus functions on stimulus equivalence class formation: Implications for understanding and treatment of anxiety. European Journal of Behavior Analysis, 10(2), 215-234.

Watt, A., Keenan, M., Barnes, D., \& Cairns, E. (1991). Social categorization and stimulus equivalence. The Psychological Record, 41, 33-50.

Wicker, A. W. (1969). Attitudes versus actions: The relationship of verbal and overt behavioral responses to attitude objects. Journal of Social Issues, 25, 4178. doi: 10.1111/j.1540-4560.1969.tb00619.x

Recebido em: 09/04/2016 Primeira decisão editorial em: 06/07/2016 Aceito em: 13/09/2016

\section{Notas:}

* Endereço para correspondência: Universidade Federal de São Carlos, Departamento de Psicologia, Laboratório de Estudos do Comportamento Humano (LECH). Rodovia Washington Luis, km 235, Caixa Postal: 676, São Carlos, SP, Brasil; CEP $13565-905$.

1 O termo transferência de funções é utilizado desde a década de 80 para descrever a extensão das funções de um estímulo para outro(s) que participa $(\mathrm{m})$ de uma mesma classe de equivalência, sem a necessidade de exposição deste(s) estímulo(s) às contingências de reforçamento diferencial. Apesar de o termo "transferência de funções" ser amplamente utilizado nas literaturas nacional e internacional, alguns consideram seu uso questionável, uma vez que o termo transferência expressa, literalmente, não um compartilhamento de funções ou extensão do controle para outros membros da classe, mas, como o próprio nome diz, um deslocamento de determinada função de um estímulo para outro. Alguns pesquisadores não utilizam tal termo, como Sidman (1994), que emprega o termo "fusão de classes" (class merger) para se referir à essa extensão do controle de emissão de respostas, e Bennett, Meulders, Baeyens \& Vlaeyen (2015), que utilizam o termo "generalização simbólica". Neste artigo optamos por manter o termo utilizado na maioria dos estudos da área.

A pesquisa contou com o apoio financeiro da FAPESP e CNPq. 